\title{
Protée
}

\section{L'allégorie : image insue et geste créateur}

\section{Francesca Caruana}

Volume 33, numéro 1, printemps 2005

\section{L’allégorie visuelle}

URI : https://id.erudit.org/iderudit/012268ar

DOI : https://doi.org/10.7202/012268ar

Aller au sommaire du numéro

Éditeur(s)

Département des arts et lettres - Université du Québec à Chicoutimi

ISSN

0300-3523 (imprimé)

1708-2307 (numérique)

Découvrir la revue

Citer cet article

Caruana, F. (2005). L'allégorie : image insue et geste créateur. Protée, 33(1), 67-76. https://doi.org/10.7202/012268ar

\section{Résumé de l'article}

La peinture ne manque pas de figures allégoriques. Leur interprétation, marquée par l'histoire et l'histoire de l'art, ne parvient pourtant pas à masquer l'importance de l'acte créateur qui préside à leur existence. C'est cette dimension trop vite classée dans l'habitude esthétique que cet article propose d'explorer, à partir du fait que toute forme laissée par l'artiste ne rend finalement compte que d'une métaphore du for intérieur : le geste créateur apparaît alors comme l'allégorie du musement. d'utilisation que vous pouvez consulter en ligne.

https://apropos.erudit.org/fr/usagers/politique-dutilisation/ 


\section{L'ALLÉGORIE: IMAGE INSUE ET GESTE CRÉATEUR}

FRANCESCA CARUANA

Dans le domaine des arts plastiques, l'allégorie occupe une place privilégiée. Dans cet article, je m'intéresserai plus particulièrement à l'art contemporain qui, par l'usage de multiples médias, rend les figures allégoriques particulièrement visibles.

En effet, si, de l'incontournable allégorie de la caverne fouillée par la rhétorique de l'Antiquité, nous passons sans difficulté à l'allégorie comme l'une des formes esthétiques, cela suppose, d'une part, de ne pas restreindre la figure à son expression discursive ou, tout au moins, de la préciser, mais aussi d'insister sur le rôle plus particulier qu'elle tient dans les arts plastiques. L'art traditionnel présente sous ses formes les plus complexes cette mise en présence d'une idée abstraite par une représentation concrète. L'idée est soutenue, dirait-on, par un ensemble de figures propres à livrer sa signification. Un ensemble de formes substitutives dont l'analyse vérifie la valeur symbolique, à condition qu'elles soient répétées et que les formes perçues le soient en tant que code: une balance sera la figure de la justice, le serpent, l'élément de la discorde, tout comme l'Amour, qui, au moins depuis le XVII e siècle, a besoin de nudité et d'enlacement pour ne pas être interprété comme une image de la création ou du péché originel. Comme le présente le Groupe $\mu$, l'allégorie est "faite de métaphores", mais il faut disposer du «code secret» (Groupe $\mu$, 1982: 137). Ce ne sont pas tant les éléments symboliques mis en jeu qui comptent dans l'évaluation allégorique, mais la mise en scène d'un ensemble d'éléments dont l'interprétation en contexte élargit la signification habituelle.

L'idée développée dans cet article est d'étendre l'allégorie au geste créateur et de montrer que toute forme surgie de l'artiste est une allégorie ${ }^{1}$, une allégorie du musement ${ }^{2}$; cependant, il ne s'agit pas de figures appartenant à un code, mais à un processus analogique par lequel elles émergent, ce qui implique de l'allégorie qu'elle soit subordonnée à des formes catégorielles. Elle est elle-même, dans la théorie sémiotique de Peirce, une figure troisième de l'icône, «interprétante» d'une simple image; elle sera ici trichotomisée à son tour pour discerner les formes de l'émergence. On peut comprendre cela, sachant que l'allégorie est une icône, plus précisément une hypoicône, et, qu'à ce titre, les figures du musement relèvent 
de la priméité; le processus par lequel une forme naît de la main du créateur est connu rétrospectivement par rupture du musement et ne saurait être symbolique. Il ne saurait donc y avoir de symbole du musement. En ce sens, l'argumentation portera sur ce en quoi l'allégorie est une figure implicite du musement et dira en quoi elle se différencie, d'une part, de l'allégorie picturale traditionnelle et, d'autre part, comment elle est une réponse insue aux pressions de coupures instaurées par la relation musement/réalité.

Lorsque Delacroix ou Courbet donnent une version de la Liberté ou de la Vérité, ils utilisent des formes susceptibles d'être comprises par le public: appropriation d'une gestuelle révélatrice pour la liberté qui brave l'avenir, vision de l'atelier comme lieu de la vérité chez Courbet, au sens d'une signification unique qui se veut être authentique, réaliste, etc. Le processus associatif par lequel ces artistes usent de figures déjà connues rend possible l'accès à l'œuvre. L'œuvre est armée pour son interprétation. Il n'en est pas de même pour les œuvres contemporaines, qui peuvent aussi bien aborder de tels «sujets» sans utiliser pour autant les codes prévus. «La vérité changera l'art» de Ben (ill. 1) conserve sa dimension allégorique en ne faisant appel à aucune représentation rhétorique langagière, mais propose (ou impose) une approche de l'art qui, du seul fait d'être perçue comme art, ne peut qu'en envelopper la fonction allégorique.

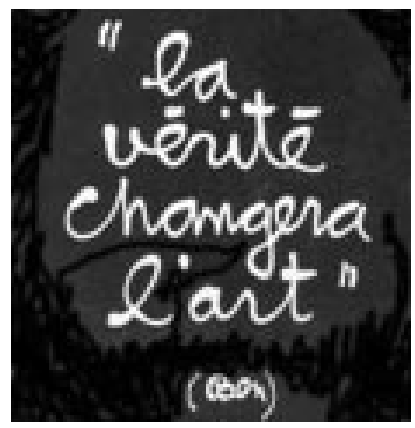

Ill. 1. Ben, La vérité changera l'art, s.d. (C) Ben/sOdRAC (Montréal) 2005.

L'objet de cette sémiose est posé comme étant la vérité, vérité d'un signe dont le mode d'existence est le tableau, mais ce mode peut être tout autre chose dans laquelle l'expérience analogique de la vérité a été faite. Toutefois, le signe qui est perçu dans le tableau renvoie à l'interprétant qui établit la relation entre l'objet et le signe représenté. L'analogie présentée est une forme allégorique de la vérité en ce qu'elle montre in situ le changement annoncé, mais emploie, pour ce faire, une écriture dont la reconnaissance efficiente appartient à un autre domaine, celui du langage. Le langage n'est pas la peinture, il en use métaphoriquement pour illustrer une fonction de l'art.

\section{LES FIGURES ALLÉGORIQUES DANS LA TRADITION}

Le terme d'allégorie est couramment employé pour désigner une chose par l'intermédiaire d'autre chose, ou le passage de l'abstrait au concret. C'est ce qu'illustre le très célèbre tableau de Delacroix, dont l'allégorie de la liberté ne peut s'aborder qu'en tenant compte de son contexte historique.

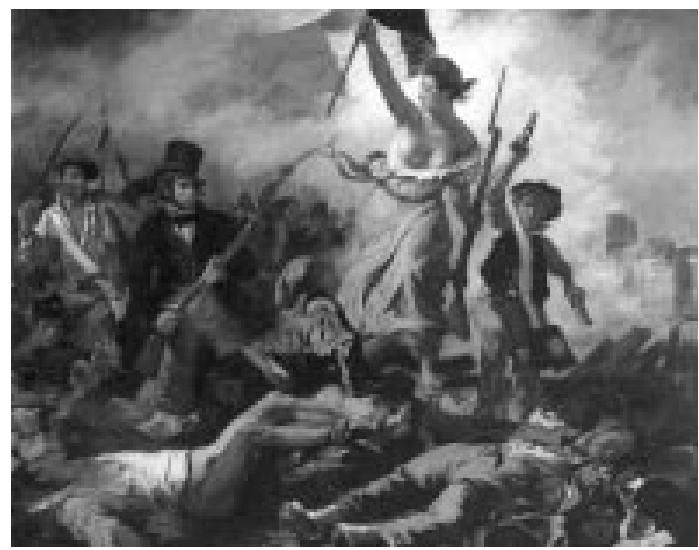

Ill. 2. E. Delacroix, La liberté guidant le peuple (1830).

Delacroix, en rupture avec la période classique, use de toutes les valeurs passionnelles, sentimentales, où la fatalité et la mort se rapportent à la nouvelle culture romantique. Le tableau montre une image du peuple guidé par une femme debout, marchant sur des morts, affublée du bonnet phrygien, et à demi dénudée. Le drapeau bleu, blanc, rouge, symbole de la révolution de 1789, devient une sorte de bouclier contre la mort, occupant le haut de la composition triangulaire et la 
clôturant. Les éléments de la composition passent du niveau iconographique des morts à celui plus éthéré d'un symbole de liberté (du légisigne symbolique) ${ }^{3}$. Le personnage féminin, disproportionné par rapport à la réalité, est la figure allégorique de la liberté, constituée elle-même de symboles admis par la tradition et détournés à d'autres fins: l'habitude veut que la femme ne soit pas sur les barricades, qu'elle soit vêtue et ne se mêle pas aux morts puisqu'elle ne guerroie pas. À la faveur d'une inversion symbolique, le personnage devient une icône à partir de laquelle chacun peut déduire des significations que Panofsky qualifierait d'implicites ${ }^{4}$. Le bref descriptif de ce tableau montre comment les symboles contribuent à permettre au spectateur d'inférer «les éléments composant la cible du parallélisme qui structure l'objet du signe» en proposant une icône ${ }^{5}$. Les éléments, tels que le drapeau, sont reconnaissables et perçus en tant que symboles; ces mêmes éléments présents sur le mur d'un hôtel de police endosseront un sens opposé: le drapeau représentera la loi, le lieu même de la loi et de son application; la valeur du drapeau passe d'un symbole de liberté (au sens de l'affranchissement de l'ennemi) à la réduction des libertés, nécessaires au

fonctionnement d'une communauté. La présence d'un drapeau sur une telle façade n'a jamais été le symbole d'une révélation libératrice ou militante. Dans ce type d'exemple, l'attribution symbolique attachée à un drapeau ou à un tableau pour signifier liberté ou peinture dépend d'une certaine disposition de la part du spectateur qui doit en partager les règles. Comme le défendait Panofsky, le registre iconologique fonderait un contenu intrinsèque (1967 : 30-31). Le sens d'une telle assertion ne reste acceptable qu'à la condition de ne pas réduire l'interprétation à un sens caché qui serait révélé dans les formes. Il s'agit donc de prendre en compte une configuration de signes dont on ne peut rien dire a priori, qui ne représente rien de ce que l'objet représenté est censé montrer, mais qui maintient une signification possible produite par convention.

La question est de savoir quels sont les éléments signifiants pour l'artiste au moment de la réalisation de l'œuvre. La figure allégorique prend ici une valeur essentiellement historique. Il est peu probable que des artistes usent de tels codes aujourd'hui pour faire passer un idéal de liberté. Il serait plutôt déplacé vers la transgression des supports (inscriptions murales sans souci de surface conventionnelle, graffiti, interventions dans la rue, etc.) ou la référence au langage, comme le fait l'artiste Ben. Cela signifie que, tout en étant constituée de symboles, l'allégorie n'est pas un symbole; elle rend compte avant tout d'une composition d'éléments qui, ensemble, forment un dispositif propre à figurer des qualités, indicibles autrement.

L'allégorie prend une tout autre dimension en sémiotique. La théorie de Peirce accorde une place privilégiée à la notion d'icône, qui est détaillée, trichotomisée en images, diagrammes, métaphores, c'est-à-dire en hypoicônes, dimension de l'icône précisant la place de l'allégorie. L'allégorie va se situer du côté de la métaphore. Le déplacement apparent, justifié par l'explication rhétorique, peut faire admettre qu'elle soit de l'ordre de la métaphore. L'évidence n'est pas pour autant acquise. $\mathrm{Si}$ déplacement il y a, encore faut-il le comprendre au sens trivial du terme, dans la mesure où la psychanalyse lui attribue plutôt la notion de condensation, susceptible de modifier la conception de la métaphore pour se rapprocher du geste créateur. Il resterait à prouver que tout acte de création relève de l'histoire personnelle, ce qui conduirait à une investigation trop large. Toutefois, on prendra en compte l'aspect individuel du créateur comme coupure lorsque l'œuvre naît, sans penser que la métaphore puisse se réduire à un déplacement d'images. Il y a donc plusieurs termes à préciser, dont l'articulation s'avère essentielle: il s'agit d'hypoicône, de métaphore et de leur contexte d'émergence. Lorsque Peirce énonce le contenu de ce qu'il entend par hypoicône, il le présente comme une nécessité théorique visant à ne pas identifier les représentations que nous percevons, surtout quand elles ne peuvent être immédiatement interprétées en tant que représentations ${ }^{6}$. Le cas d'un tableau offre l'exemple même de l'hypoicône sans la désignation du "cartel» 
qui en fait un signe. C'est le nom du «representamen iconique: tout tableau indépendamment de son mode de représentation, sans légende ni étiquette, est une hypoicône" (Deledalle, 1979:20). Il reste que l'hypoicône est un representamen dont la qualité première «en tant que chose» est d'être un substitut d'une autre chose (à laquelle elle ressemble pour maintenir la logique théorique). Il en sera question plus loin. Auparavant, le terme de métaphore mérite d'être précisé. L'idée fréquemment rencontrée l'assimile, de façon définitoire, au symbole et soulève souvent celle de déplacement. La confusion des termes aboutit parfois à une erreur d'interprétation:

[...] il s'ensuit que les conceptions de Peirce et de Panofsky sont faibles au même endroit: l'iconologie ne s'adapte exclusivement qu'à la catégorie de la peinture symbolique; la sémiotique présuppose que toute peinture est, en dernière analyse, symbolique. Elles échouent donc communément devant l'évidence que les différents genres picturaux sont inégalement déterminés vis-à-vis de la finalité cognitive, que les moins symboliques d'entre eux (la nature morte par exemple), non seulement résistent à l'intellectualisation mais ne semblent viser d'autre effet final que l'iconicité. (Château, 1997: 61)

L'imprécision commise dans le non moins remarquable ouvrage de D. Château est d'attribuer à l'icône une fonction indicielle, dans l'innocuité générale de la garantie qu'offre pourtant la hiérarchie des catégories. Il ne s'agit pas d'orthodoxie sémiotique mais de logique, et l'enfermement critique évoqué n'autoriserait l'icône qu'au travers d'un préalable perceptuel dont l'interprétation iconique ne serait qu'une reconnaissance. L'icône ne peut être reconnue, c'est le propre même de ses qualités formelles, elles sont muettes; si tel n'était pas le cas, il s'agirait d'un indice renvoyant à des relations de contiguité avec leur objet. À quel type d'objet renvoie la Liberté évoquée par Delacroix? À quel type de relation de contiguité? Peirce permet d'éclaircir la question en plaçant la métaphore au troisième degré d'hypoiconicité. Il s'avère dès lors utile de pénétrer de manière plus approfondie ce que l'on entend par iconicité, à savoir l'approche d'un système de représentations non interprétées; mais l'ensemble des travaux réalisés sur ce sujet permet d'utiliser le concept sans avoir à le redéfinir dans ce contexte, sachant qu'il englobe plus largement les spécificités de l'icône. La métaphore est un degré spécifique de l'image muette 7 qui va promettre des significations par procuration et des figures analogues. La métaphore va concerner aussi bien l'image produite que le jugement de perception qui lui est afférent.

On peut en gros diviser les hypoicônes suivant le mode de priméité auquel elles participent. Celles qui font partie des simples qualités ou premières priméités sont des images; celles qui représentent les relations, principalement dyadiques ou considérées comme telles, des parties d'une chose par des relations analogues dans leurs propres parties, sont des diagrammes; celles qui représentent le caractère représentatif d'un representamen en représentant un parallélisme dans quelque chose d'autre, sont des métaphores. (2.277)

(Peirce, 1978: 149)

Nantie de ces définitions sémiotiques, l'Allégorie de la prudence de Titien peut être observée en tenant compte du fait que le codage est nécessaire au suivi des transformations minutieuses auxquelles s'attache Daniel Arasse dans son analyse (1997: 108-110).

Dans la tradition iconographique, la fonction première attribuée aux éléments de représentation, tels que le visage tricéphale, était de montrer les trois âges de la vie. Mais Titien y fait aussi son autoportrait, celui du vieillard placé à gauche, légèrement en retrait, ce qui pour un autoportrait n'est pas très "orthodoxe». C'est précisément cette position de l'autoportrait que D. Arasse établit avec le tricéphale animal.

Titien a superposé une image démodée de la Prudence (les trois têtes humaines d'âge différent) à une image moderne de la même Prudence (le tricéphale animal du "monstre Sérapis en buste»).

(Ibid. : 107)

Titien introduit un trouble dans le codage, dont Arasse proposa d'abord qu'il puisse avoir son origine dans les règles de l'ars memorix, «image-souvenir efficace, léguée en tant que telle par Titien à son clan, dont les membres ne pouvaient manquer, ne 


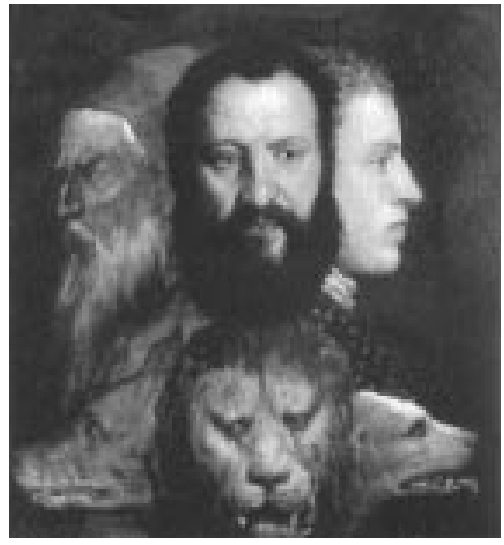

Ill. 3. Titien, Allégorie de la Prudence, 1530. National Gallery de Londres.

l'oublions pas, de reconnaître immédiatement les figures", la mémoire étant de plus une partie de la Prudence (ibid.: 108). Il suffit de se rappeler les travaux de Giordano Bruno sur l'art de la mémoire et les opérations de transfert entre "le monde intérieur de l'imagination aux étoiles» et «le monde céleste à l'intérieur de l'homme" pour saisir l'incidence de la mémoire dans la forme allégorique (cité par Yates, 1975: 209). En tout état de cause, il m'importe d'insister sur le fait que Titien, déjà, introduit une sorte de dissidence dans les dispositifs habituels de représentation et convoque les pratiques de l'art de la mémoire (ce sera un repère important pour le débat contemporain). Les modifications qu'apporte ainsi Titien par la présence du tricéphale animal sont interprétées aujourd'hui par Arasse comme une "condensation figurative»:

[...] figure totémique où la mort s'inscrit dans l'ombre de sa figure absente, le double tricéphale est aussi le terme marquant l'accession à la plénitude des trois Temps fixés en un et à l'immortalité de l'artiste en son ceurre. (1997: 108)

L'étude approfondie par Arasse met l'accent sur une prise de position inférentielle de la part du peintre. La figure allégorique rend compte aussi de l'intimité de l'artiste pour qui la toile est une scène où sa propre histoire se joue autant que celle des symboles.

Le fait que ces codes soient dérangés a priori n'entame en rien les significations traditionnelles; ce qui les modifie, c'est la juxtaposition des deux et surtout les conditions dans lesquelles le déplacement est opéré, ce qui revient à assimiler la métaphore au déplacement (notons au passage que Daniel Arasse parle clairement de condensation figurative). Dans un premier temps, je m'accorderai avec d'autres sur l'idée de déplacement, mais lorsque la place personnelle, individuelle aura été mise en relief, il faudra conclure que seule la condensation, au sens psychanalytique du terme, représente la métaphore produite. La tradition use d'images connues, dont le sens est mis en scène avec des façons de faire différentes. Un artiste comme Titien use de ces codes pour les enrichir et les déjouer ou jouer sur ce que l'homme devrait être, une sorte de figure idéalisée des comportements sociaux. En cela, il se décale de l'orthodoxie générée par l'usage de tels signes. Bronzino, par exemple, respecte la nudité du modèle et l'enlacement des corps pour signifier l'allégorie de l'Amour, il ne revient pas sur ces signeslà en les transposant ou en les interprétant. Si quelque chose change du point de vue de l'iconicité, puisque l'interprétation ne peut se faire qu'au prix d'une réactualisation du décodage, cela ne change rien au processus. Il reste en effet que l'artiste produit une image composite faite des qualités formelles internes ou mentales et de qualités formelles physiques, constitutives de l'image possible. Ce point de vue pourrait convenir à $\mathrm{D}$. Château et à sa critique de l'icône peircienne, mais les stades de l'icône qu'il propose renforcent malheureusement l'idée d'une image en soi, qui relève du contexte intrinsèque, et d'une image plus réelle, qui dépendrait d'un contexte extrinsèque; images qui seraient implicitement pourvoyeuses d'indices (Château, 1997: 59-60). Le processus de formation de l'allégorie est une forme «consolidée» de l'icône, au sens où sa nature composite rend compte du rapport du signe à l'objet immédiat et condense ses trois modes de présentation (descriptif, indicatif et copulant):

Les objets dans les signes peuvent être présentés comme de simples idées, telle une notion absolument définie ou distincte de toute autre. Ils peuvent être présentés comme requérant brutalement notre attention. Ils peuvent en dernier lieu s'adresser à notre raison, ou se présenter comme des habitudes que nous avons d'ores et déjà acceptées. (8-349)

(Réthoré, 1988: 414-422) 
Pour le dire simplement et montrer la détermination du signe par l'objet sur l'interprétant, il suffira d'observer toute œuvre qui assure le passage de sa potentialité à son existence (si toutefois elle est conçue, mais je penche pour dire qu'elle est plutôt prise dans le musement).

L'hypothèse qui suit, et que je propose comme une réponse au doute de $\mathrm{D}$. Château sur l'efficience de l'icône peircienne, prend en compte précisément la dimension allégorique de l'icône, comme stade interprétant de la métaphore. Si indicialité il y a, comme il l'affirme, elle se situe alors dans la relation entre texte et image que fonde le titrage des tableaux par les peintres. Le titre assure ainsi la source allégorique, qu'elle soit mythologique (Le Jugement de Pâris de Poussin), rhétorique (Je ne sais pas peindre de Ben) ou formelle (Composition VIII de Kandinsky), en préservant toujours l'hypoicône d'une norme qui la précéderait. Cela revient à trichotomiser modestement l'allégorie, puisqu'elle montre d'abord la médiation (idée troisième de l'allégorie, il serait plus juste de parler de dégénérescence de la tiercéité de la métaphore) que représente le titre entre le niveau symbolique discursif et l'image, par une configuration diagrammatique possible entre mots et image. Puis elle montre l'action réellement diagrammatique de la proposition rhétorique (idée de second) qui, dans le cas de Ben, va du mot au sens et du sens à la déduction, il apporte en quelque sorte la preuve de l'assertion dans un domaine où le vérifiable n'est pas un préalable! Enfin, l'allégorie montre le sentiment premier, lié à la simple mise en place de figures dans l'image, qu'elles soient figuratives ou non (comme chez Kandinsky). Ces trois niveaux allégoriques sont présents dans la peinture de Poussin, par exemple dans Pyrame et Thisbé (1648).

On ne peut pas comprendre une peinture de Poussin comme un simple paysage, dès lors qu'il est intitulé Pyrame et Thisbé. Paysage ou personnages? L'allégorie réside dans la relation de réversibilité (gouvernée cependant par le jeu de langage contenu dans le titre), elle est induite par les éléments formels qui produisent, quoi qu'il en soit, une icône d'un type particulier: une métaphore plastique qui tient lieu de paysage et un diagramme (langagier) qui le théâtralise pour dénoter son ancrage mythologique. Rien a priori ne vient éclairer cette relation, il n'y a pas de transparence entre texte et image. On pourrait aller jusqu'à dire que l'image muse seule pendant que le titre en inscrit les termes. Cette inscription picturale est l'outil du repérage de la subjectivité transmise par les images et dévoile comment, dans le sujet énoncé, transparaît le sujet de l'énonciation.

\section{ALLÉGORIE ET GESTE CRÉATEUR}

Ce qui vient d'être dit énonce plus techniquement ce que la peinture contemporaine fait perdurer, non pas de la tradition, mais de la continuité créatrice. Contextualiser le geste créateur dans le domaine psychologique de la subjectivité reviendrait à faire de l'artiste un individu, nié de fait par les interactions sociales dont il dépend. Il en est de même pour la production des œuvres et au-delà des images qui les constituent. Ces images sont de nature prédicative, ce qui permet de nuancer certains degrés de l'allégorie: - L'allégorie - en utilisant des métaphores issues d'images toutes prêtes à servir un discours - établit un parallélisme par contiguité dans un code langagier ou plastique propre à la charger de sens.

- Lorsque l'allégorie autorise une part interprétante du spectateur, c'est-à-dire en permettant au spectateur, par inférence abductive, d'imaginer une source ou de la mettre en parallèle avec une proposition plastique plus injonctive, elle établit alors une relation de ressemblance dans ce parallélisme.

- Enfin, lorsque l'allégorie est insue et que les images se forment sans que le répertoire de références puisse vérifier ou proposer lui-même une source, le champ est alors si vaste qu'on peut dire qu'il est à lui seul la figure allégorique du musement de l'artiste. Il perd toute valeur prédicative si ce n'est a posteriori, lorsque l'interprétation possible en aura été faite. Dans cette attente, on a affaire au niveau le plus bas de l'allégorie, un niveau de priméité, où toute image révélée, surgie, issue de la main de l'artiste peut ne pas être indexée à un répertoire. 
Bien sûr l'allégorie sera contestée du point de vue formel, puisqu'on peut toujours répertorier une œuvre dans l'univers figuratif, abstrait ou selon la dualité moderne/contemporain, etc. Quoi qu'il en soit, ce qui est pris en compte ici, c'est la capacité de l'artiste à produire des images qui, libres de toute référence ou indexées à certaines, se présentent comme la figurabilité du processus créateur. En effet, quand bien même un artiste répondrait à une thématique par une figure allégorique connue, la manière dont il l'interprète, lorsque l'allégorie est codée, est une posture esthétique discriminée de toute autre, sa réponse reste singulière et, de ce point de vue, le musement à l'œuvre, dans cette sémiose, a pour objet la présence d'images prototypiques convergentes autour de légisignes acquis par l'histoire de l'art ${ }^{8}$. Le savoir de cette sémiose "musée» est insu, mais tissé dans l'histoire personnelle de l'artiste. Ce qui le fera tendre vers les signes d'une figure déjà connue ou reconnaissable. Ainsi, on peut préciser deux dynamiques dans ces sémioses, la première produite par rupture avec les éléments formels, prototypiques, ayant pour objet le rapport entre l'artiste et son musement, et une autre plus «consentie», dont l'objet montre un savoir sur les représentations. Le répertoire allégorique relève de ces deux types à la fois, mais en formulant le terme de répertoire, j'aborde un des niveaux de l'allégorie, celui dont les représentements 9 ont une dimension symbolique, qui nous feront toujours reconnaître le Printemps dans une toile, qu'elle soit de Botticelli ou de Poussin.

Cette création possible, qui est d'une nature particulière, peut être rapprochée de ce qu'Adorno nomme «allégorie de l'improvisation», à propos de la musique informelle. L'idée est séduisante car elle s'applique au moins aux deux formes énoncées, la première concernant la forme allégorique "orthodoxe», qui s'apparenterait à la catégorie de la «récurrence modifiée», et la deuxième réduisant la musique informelle à l'allégorie de l'improvisation ${ }^{10}$. Le musement est le tissu même de l'improvisation et de l'application. L'importance du musement pourrait tenir lieu de subjectivité, si on ne prenait la précaution de dire qu'il est social, "collectif ", c'est-àdire comme flot continu d'un groupe ayant en commun les mêmes habitudes.

Le travail du scribe est supposé se terminer avant que le travail de création ne soit accompli. Le scribe est supposé savoir lire les pensées (et là nous pourrions le considérer comme Manticien) à tel point qu'il connaît une partie (peut-être le tout) du travail créateur du Museur jusqu'au moment présent, mais non ce qui est à venir. Ce qu'il veut que le graphe exprime concerne l'univers tel qu'il sera quand il viendra à l'existence. S'il hasarde une assertion pour laquelle il ne trouve pas de garantie dans ce que le Museur a pensé jusque-là, elle peut ou peut ne pas s'avérer vraie. $(4.431)^{11}$

De cette proposition, la sémiose de création produite est une inscription de même type que la mantique mythologique: tout se passe comme si cette sémiose traduisait un courant du flot de pensées:

[L]a sémiose propose à l'analyse de son processus un représentement, un objet dont il tient lieu et un interprétant en charge de "livrer", dans l'assertion, une information sur l'objet et l'identification de ce dernier [...]. Le représentement iconique se prend lui comme objet. 12

Cela ne signifie pas que le musement soit unilatéral, marqué par les affres géographiques des lieux où vivent les artistes; pourtant cela reste en partie vrai. Ce qu'il faut retenir, c'est que la formation même de l'hypothèse créatrice se fait dans un certain contexte et qu'un Mélanésien de Nouvelle-Calédonie n'aura pas la même qualité de musement qu'un Provençal: ce qui était déjà présent dans le modus agendi, scribendi ou tractandi des Métamorphoses d'Ovide, qui met en valeur les qualités stylistiques et rhétoriques propres aux divers champs culturels.

L'allégorie a besoin d'éléments symboliques pour être saisie comme telle. Or les figures du musement ne peuvent être symboliques. Ce que l'on peut dire, c'est que la nécessité du surgissement met en place des signifiants et, de ce point de vue, assure une fonction symbolique. Ces formes sont un «précipité»du musement, des signifiants «mis pour» qui représentent une stase du flot de pensées. Partant du fait que 
l'allégorie est la représentation concrète d'une figure abstraite, on considérera le flot du musement comme une suite continue d'abstractions, au sens visuel, émotionnel, réel, etc. Autrement dit, la virtualité des images du musement doit être comprise comme une abstraction; ces images ne sont pas réelles mais, en produisant certains effets, elles acquièrent une dimension de réalité. Elles peuvent être chaotiques, parfois violentes, parfois apaisantes, continues, subordonnées ou pas aux conditions physiques vécues par le sujet musant, mais elles sont omniprésentes, quel que soit le moment de création. L'artiste, face à la pièce qu'il est en train de réaliser, muse sur son passé ou sur le prochain événement plastique, et alors qu'il est tout à son œuvre, et tout en continuant à muser, des formes se détachent de lui, «tombent» en quelque sorte sur sa toile ou son support, et s'oublient. Ces formes-là, découpes dans le processus, sont une allégorie de ce que peut être le moment musé de la création dont on sait bien, qu'en dehors de cette émergence, il reste totalement inaccessible. Les limbes de l'œuvre agissent comme un lapsus ou un acte manqué, seuls à rendre compte d'un «agir» de l'inconscient. Ces représentations, «déhiscences» du geste créateur, comme aurait pu l'affirmer MerleauPonty ${ }^{13}$, sont des icônes, elles ne peuvent être ni des images, ni des diagrammes, puisqu'elles sont tributaires d'un inventaire de conditions qui ne montrerait aucune pertinence matérialisée de l'intérieur, ni exclusivement des métaphores, puisque les déplacements ou condensations opérés nécessiteraient un codage identique pour tous. L'établissement de ces codes par la tradition ont ici un sens réel, si l'on souhaite figurer des énoncés tels que liberté, justice, mort, amour, etc.

La description de ce phénomène permet de concevoir que toute création est allégorique par une inévitable sédimentation processuelle: il s'agit d'une image du musement, d'une ab-straction, puisqu'elle n'a pris forme qu'en tant que virtualité surgie, et qu'elle est représentée de manière concrète. De cette manière, aucun indice ne vient brouiller le flot du musement, si ce n'est la rupture. On ne peut connaitre le musement que par coupures. Ainsi, toute forme produite ne peut être qu'une abstraction du musement, inaccessible concrètement parlant. La forme qui en ressort est nécessairement la concrétude d'une image de priméité produite dans le musement ou elle est ce que Daniel Arasse, sur le mode comparatif d'avec la psychanalyse, pourrait nommer la figurabilité de l'œuvre (1997: 14, note24).

\section{L'ALLÉGORIE: PHASE D'UNE RELATION} ENTRE MÉMOIRE ET GESTE CRÉATEUR

Dans les arts dits primitifs, ce qui compte, c'est de restituer un «savoir» sous forme symbolique, d'en garder la mémoire en en préservant les signes. Ces signes-là sont des signes diacritiques. Ils rappellent ceux de la tradition par leur structure, mais pas forcément par imitation. Ainsi l'installation Les Tarots qui pleurent, de l'artiste kanake Denise Tiavouane, reprend, de manière critique et poétique, les éléments de la culture locale (le tarot est un tubercule voisin de l'igname que l'on trouve sur la Grande-Terre en Nouvelle-Calédonie) en installant, dans un champ, des bâtons mis à leur place, "vêtus» de jupe de paille, sonorisés et animés dans une petite chorégraphie qui reste sur place. Elle dénonce ainsi la blessure coloniale qui a atteint les Kanaks dans leur quotidien. Chacun sait pourtant que, sans imiter ni les légendes, ni les formes traditionnelles, ce répertoire de signes (elle attribue ici aux tarots la même valeur sacrée qu'a l'igname, mais en symbolisant l'agriculture qui est indispensable à l'harmonie entre tradition et quotidien) est une proposition concrète (plastique) de significations communes affirmant leur attachement à la culture kanake.

On retrouve des phénomènes semblables dans l'art contemporain, où l'analogie formelle suffit à réactiver la mémoire de signes dont on ne connaît pas vraiment la signification, mais qui en «honorent» d'autres. La singularité de ces travaux est de proposer une imitation virtuelle. Ce qui est imité est une vague ressemblance à un objet dont le modèle ne peut être vraiment contrôlé, mais dont tout le monde s'accorde sur la validité de mémoire. Il s'agit d'une ressemblance au discours. 


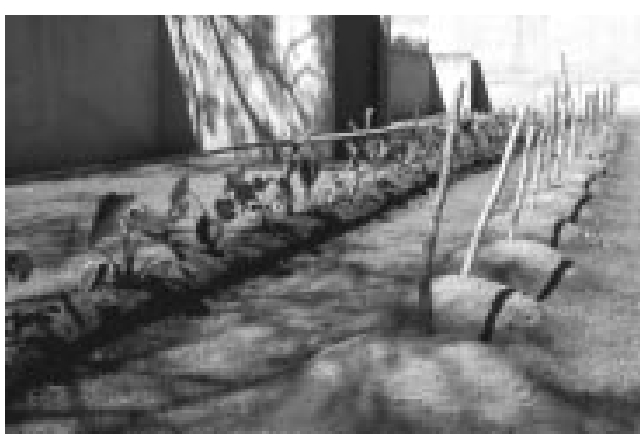

Ill. 4. Denise Tiavouane, Les Tarots qui pleurent. (C) Centre culturel Jean-Marie Tjibaou.

Les objets de l'enfance font partie de notre oubli, l'installation d'artistes réactive la mémoire qu'il en reste et actualise des images qu'on ne se savait plus posséder. Il s'agit alors de souvenirs que l'organisation, le mécanisme de mémoire fait surgir. La mémoire nécessite une organisation. On ne se rappelle pas de tout.

Or, la sélection qui s'opère dans le répertoire des formes possibles émerge vraisemblablement selon les arcanes du désir parce qu'enfouis dans l'activité continue du musement. En ce sens, le lieu de la mémoire pour l'œuvre niche dans le désir de création. L'exemple de l'œuvre Made of the $1^{\text {st }}$ of May (1995), de l'artiste contemporain Philippe Parreno, en donne un aperçu ${ }^{14}$. La réalité est livrée tout entière par la mémoire d'un signe particulier, dont l'approximation est le mode d'être. Ainsi, ce qu'engendre l'effet de mémoire est un rapport de type poïétique et rhétorique par lequel toute œuvre, et a fortiori lorsque celle-ci comporte un titre, est une allégorie du musement. Le rôle de sentinelle sensorielle que peut tenir le désir permet l'inscription de traces, devenant plastiques au cours du processus créateur. L'œuvre tient lieu d'une instanciation mnémonique.

Ainsi l'art contemporain kanak, peut-être plus que tout autre art contemporain, utilise la mémoire comme valeur infinitive de l'émergence artistique, par addition de légendes ancestrales, du désir de les perpétuer, et de l'instanciation à laquelle elles donnent lieu par allégories interposées. La question de l'allégorie remet en cause aussi bien celle de l'autorité, selon Aristote (c'est-à-dire l'ensemble des signes diacritiques qui vont édicter la reconnaissance possible du contenu de l'image), que celle d'auteur qui correspond à celle qui retient les figures du musement non travaillées par les légisignes d'une doxa iconographique.

Le mécanisme de l'allégorie, qu'il soit appréhendé sous les aspects «fonctionnalistes» de la tradition par codage de significations ou sous la forme de révélateur du processus créateur, agit en tout ou en partie comme l'effet connotatif de dénotation collective ou personnelle. Comme l'affirmait Heidegger, «l'art est poème».

\section{N O TES}

1. Il faudrait faire une distinction entre artiste et créateur, mais ce serait hors de propos. Je me contenterai de préciser que j'emploie le terme d'artiste par facilité de langage mais qu'il convient de définir l'artiste comme celui qui produit des formes et endosse l'uniforme inscrit dans les habitudes sociales, une certaine répétition à la clef, c'est-à-dire faire une peinture figurative ou abstraite, dont la reconnaissance est acquise; le créateur sera celui qui donne libre cours au musement, qui prend donc le risque d'une rupture d'avec la reconnaissance esthétique, au-delà des anticipations qu'il peut faire. Le créateur n'a pas de programme, il fait, sans se soucier non plus de quelque notion de "beauté " que ce soit.

2. Le musement est un concept propre à Peirce, relatif aux pensées continues et non contrôlées qui occupent notre esprit. Un vagabondage continu de pensées qu'il appelle un Jeu pur (Deledalle, 1990: 174).

3. On peut dire qu'il s'agit d'un symbole abstrait, «forme dégénérée du symbole dont le seul objet est un caractère général " (représentation de la France). Le symbole renvoie à l'objet qu'il dénote en vertu d'une loi, d'ordinaire une association d'idées générales (Deledalle, 1979: 25). 4. Voir les trois niveaux de significations dans Panofsky (1967: 30-31). 5. "Elle se distingue par conséquent de la métaphore filée moins par sa forme - il s'agit de la métaphore au sens formel peircien dans les deux cas - que par sa portée car la structure de l'allégorie est coextensive avec l'ensemble du texte, et non avec une de ses parties". Voir le remarquable article de T. Jappy où sont développées avec précision les distinctions entre les degrés d'iconicité de la métaphore et de l'allégorie (2002 : 85-110). 
6. Ne peut être identifié comme représentation une forme ou un son ou tout autre chose dont on ne peut dire que les qualités formelles. Le signe limité à cette présentation est admis comme signe iconique, ou representamen d'une autre sémiose. «Un representamen par la priméité seule ne peut qu'avoir un objet semblable» (2-276) (Peirce, 1978:149).

7. Le terme d'image muette reprend l'idée de sa non-transitivité; sans autre précision, elle ne dit rien en elle-même. Seule sa contextualisation permettra d'en dire quelque chose.

8. Le terme "type" vient du latin typus qui signifie "modèle, symbole», et du grec tupos qui signifie «figure, image, modèle, empreinte, marque, caractère d'écriture" (voir Dictionnaire étymologique Larousse [1971] et Petit Robert de la langue française [1990]). La trichotomie du signe en sémiotique fait apparaître le rôle fondamental du type (ou légisigne) comme signe de loi qui gouverne une habitude de représentation (Deledalle, 1990: 150-151).

9. Le terme de représentement a été "francisé " par M. Balat en remplacement du representamen de Peirce (Balat, 2000a: 22; Deledalle, 1979: 65-66)

10. À ce propos, Adorno pense que l'absence de notation musicale ancre l'improvisation dans l'allégorie qui en est la réduction créatrice motivée aussi bien par la matérialité de la forme que par le sujet. Cité par M. Ratté, 1998 (Adorno, 1976: 246)

11. M. Balat cite une traduction de Janice Deledalle-Rhodes dans laquelle se distinguent deux niveaux de savoir (Balat, 2000b: 138).

12. M. Balat développe ces trois positions: le manticien, la pythie et l'interprète. Ces trois concepts illustrent trois positions interprétantes d'un objet qui se veut inouï au sens de l'impossibilité a priori d'en savoir quelque chose. Tout ce que l'on en sait et saura passe par la forme propre de sa représentation (ibid.: 142).

13. On peut rapprocher cette rupture du musement, ce moment d'inscription, de ce que Merleau-Ponty décrivait comme des présences au monde rendues visibles (1986: 72).

14. Cette image de Parreno est reproduite dans l'ouvrage de Grosenick et alii (1999: 381).

\section{RÉFÉREN CES BIBLIO G RAPH IQ U ES}

ADORNO, T.W. [1982]: "Vers une musique informelle", dans Quasi una fantasia, Paris, Gallimard;

[1976]: Autour de la théorie esthétique, Paris, Klincksieck.

ARASSE, D. [1997]: Le Sujet dans le tableau, Paris, Flammarion, coll. "Idées et recherches".

BALAT, M. [2000a]: Fondements sémiotiques de la psychanalyse, Paris, L'Harmattan;

[2000b]: Psychanalyse, logique, éveil de coma: le musement du scribe, Paris, L'Harmattan.

CHÂTEAU, D. [1997]: Le Bouclier d'Achille, théorie de l'iconicité, Paris, L'Harmattan, coll. "Champs visuels".

Deledalle, G. [1990]: Lire Peirce aujourdh'ui, Bruxelles, De Boeck Université, coll. "Le point philosophique»;

- [1979]: Théorie et pratique du signe, introduction à la sémiotique de Charles S. Peirce, Paris, Payot.

Grosenick, U., L. B. LARSEN et B. RIEMSCHNEIDER (dir.) [1999]: Art at the Turn of the Millennium, Cologne, B. Taschen.

GROUPE $\mu$ [1982]: Rhétorique générale, Paris, Seuil, coll. «Essais».

JAPPY, T. [2002]: "Propos sur l'allégorie», dans T. Jappy et J. Rhétoré

(dir.), Sémiotique peircienne: état des lieux, Actes du colloque

international 2001, Canet-Plage, Presses universitaires de Perpignan.

Merleau-Ponty, M. [1986]: L'CEil et l'Esprit, Paris, Gallimard, coll.

"Folio".

PANOFSKY, E. [1967]: Essais d'iconologie, Paris, Gallimard.

PEIRCE, C.S. [1978] : Écrits sur le signe (rassemblés, traduits et commentés par G. Deledalle), Paris, Seuil.

RATTÉ, M. [1998]: "Le Problème du devenir dans le concept adornien de "musique informelle" ", dans $Æ$, vol. 3 (automne); [http// www.uqtr.ca/AE/vol_3/ratte.htm].

RÉTHORÉ, J. [1988]: La Linguistique sémiotique de Charles S. Peirce: propositions pour une grammaire phanéroscopique, thèse de doctorat d'État, Université de Perpignan.

YATES, F. [1975]: L'Art de la mémoire, Paris, Gallimard. 